POS PROCEEDINGS

\title{
Improved modeling of reactor antineutrino spectra
}

\author{
Lorenzo Périssé, ${ }^{a, *}$ Xavier Mougeot, ${ }^{b}$ Anthony Onillon ${ }^{a, c, \text { now at } d}$ and Matthieu \\ Vivier $^{a}$ \\ ${ }^{a}$ IRFU, CEA, Université Paris-Saclay, \\ 91191 Gif-sur-Yvette, France \\ ${ }^{b}$ Université Paris-Saclay, CEA, List, Laboratoire National Henri Becquerel (LNE-LNHB), \\ F-91120 Palaiseau, France \\ ${ }^{c} D E N$-Service d'études des réacteurs et de mathématiques appliquées (SERMA), CEA, Université \\ Paris-Saclay, \\ 91191 Gif-sur-Yvette, France \\ ${ }^{d}$ TUM, Physik-Department, Technische Universität München, \\ D-85748 Garching, Germany \\ E-mail: lorenzo.perisse@cea.fr
}

Over the last decades, Inverse Beta Decay antineutrino experiments conducted at short and long baselines from nuclear reactors have revealed significant discrepancies on both the rate and shape of the measured spectra compared to state-of-the-art predictions. No evidence for an experimental bias has been demonstrated, and the sterile neutrino interpretation of the reactor antineutrino anomaly is currently disfavored by recent very short baseline reactor experiments. The validity of the predictions is then questioned as the source of the observed discrepancies. This motivates a revision of the reactor antineutrino spectrum modeling, which will also be useful for a new generation of reactor experiments investigating Coherent Elastic Neutrino-Nucleus Scattering. In this context, a revisited prediction of reactor antineutrino spectra using the summation method is currently underway, including the construction of a comprehensive uncertainty budget associated to both modeling and nuclear data. The ingredients of this new prediction along with preliminary results are presented in this proceeding.

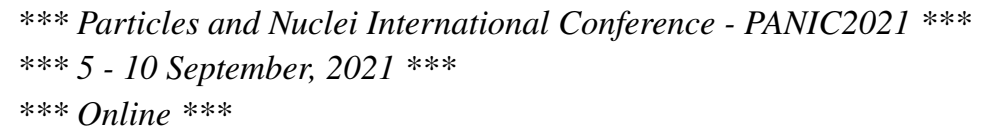

${ }^{*}$ Speaker 


\section{Introduction}

Nuclear reactors emit a huge number of electronic antineutrinos $\left(\bar{v}_{e}\right)$ originating from the fission of uranium and plutonium contained in the nuclear fuel, as well as from neutron activation of structural and fuel elements. Each fission produces two unstable fission products (FP) that undergo successive $\beta$-decays before reaching stability. About six $\bar{v}_{e}$ are emitted per fission and a reactor emits $2 \times 10^{20} \bar{v}_{\mathrm{e}} \cdot \mathrm{s}^{-1} \cdot \mathrm{GW}_{\text {th }}$. Below $10 \mathrm{MeV}$, the Inverse Beta Decay (IBD) cross-section is about $\sigma_{I B D} \sim 10^{-42} \mathrm{~cm}^{2}$ and the Coherent Elastic Neutrino-Nucleus Scattering (CEvNS) cross-section is about $\sigma_{C E v N S} \sim 10^{-40} \mathrm{~cm}^{2}$. Nonetheless, the gigantic number of $\bar{v}_{e}$ produced, associated to large long baseline or smaller short baseline detectors, balance their very low interaction cross-section. Reactor $\bar{v}_{e}$ spectra can be predicted using two methods. The summation model (SM) uses available nuclear and fission data to build a reactor spectrum based on the $V-A$ theory of weak interaction, but faces the incompleteness of databases. The second method is the conversion method. It consists in fitting a measured electron spectrum using virtual $\beta$ branches that are converted to $\bar{v}_{e}$ spectra in order to obtain the total $\bar{v}_{e}$ spectrum. The conversion method is used in state-of-the-art predictions, but several discrepancies have been observed between such predictions and experimental data. In 2011, a new evaluation of reactor $\bar{v}_{e}$ spectra was performed independently by the Saclay group [1] and P. Huber [2]. These two new predictions led to consistent deficits of about $6 \%$ statistically significant at more than $2 \sigma$ when compared to measured IBD rates of short baseline experiments, called the reactor antineutrino anomaly [3]. Furthermore, a shape difference manifesting mostly in the 4-6 MeV range between the measured IBD spectra and the predictions has been observed in several experiments, including recent ones at very short baselines. Several scenarios have been proposed to explain these anomalies. The sterile neutrino hypothesis is disfavored and detector effects are unlikely, but a misprediction with an underestimated uncertainty budget could explain them. In order to investigate the origin of these anomalies, the modeling of reactor spectra and their uncertainty budget must be revisited.

\section{Revised summation calculations}

In the SM, $\beta\left(\bar{v}_{e}\right)$ fission spectra are calculated as the sum of $\beta\left(\bar{v}_{e}\right)$ spectra of every FP $\beta^{-}$ transition. At the level of a single $\beta$-branch $b$ of a FP $f$, the associated $\beta$ spectrum $S_{f}^{b}$ is described by the $V-A$ theory of weak interaction as

$$
S_{f}^{b}\left(Z_{f}, A_{f}, E_{0, f}^{b}, E\right)=\underbrace{K_{f}^{b}}_{\text {Normalization }} \underbrace{p E\left(E_{0, f}^{b}-E\right)^{2}}_{\text {Phase space }} \underbrace{\mathcal{F}\left(Z_{f}, A_{f}, E\right)}_{\text {Fermi function }} \underbrace{C_{f}^{b}(E)}_{\text {Shape factor }} \times \text { Corrections, }
$$

where $Z_{f}$ and $A_{f}$ are the daughter nucleus charge and mass numbers, $E_{0, f}^{b}$ is the endpoint energy of the considered transition, $p$ and $E$ are the $\beta$ particle momentum and total energy. $K_{f}^{b}$ is a normalization factor such that $\int_{0}^{E_{0, f}^{b}} \mathrm{~d} E S_{f}^{b}(E)=1$. The phase space factor comes from the density of states accessible to the emitted particles. The Fermi function specifically accounts for the deceleration of the electron in the Coulomb field created by the $Z_{f}$ positive charges, and is the leading order QED correction [2]. The shape factor $C_{f}^{b}(E)$ is a modification to the Fermi function depending on the forbiddenness degree (FD) of the transition. The FD of a $\beta$ transition 
is a classification based on spin and parity changes between the parent and daughter nuclei. Shape factors are constant for transitions of the lowest FD (called allowed transitions), and are expressed as polynomials in the electron and neutrino momenta for transitions of higher FD (called forbidden transitions). Previous summation calculations used analytical Fermi functions derived for point-like daughter nuclei and constant coefficients for the shape factor polynomials. In this work, a code was developped to compute exact corrections to the Fermi functions and energy dependent shape factor coefficients for every nucleus, including the so-called nucleus finite-size and atomic screening effects. Finally, radiative corrections from QED [4] and weak current corrections (commonly referred to as weak magnetism) due to the nucleon finite size [5] are considered in Eq. 1. The $\bar{v}_{e}$ spectrum of a transition is then given by energy conservation, if recoil effects are neglected [2]. It consists in replacing the $\beta$ energy by the $\bar{v}_{e}$ energy in Eq. $1, E \rightarrow E_{\bar{v}_{e}}=E_{0, f}^{b}-E$. The spectrum $S_{f}$ of a FP is obtained as the sum of the $n_{f} \beta$-branch spectra weighted by their corresponding branching ratio $B_{f}^{b}$. The actinide fission spectra $S_{k}\left(k={ }^{235} \mathrm{U},{ }^{238} \mathrm{U},{ }^{239} \mathrm{Pu},{ }^{241} \mathrm{Pu}\right)$ are then obtained by summing the respective FP spectra weighted by their corresponding $\beta$ decay activity $\mathcal{A}_{f}$. Activities can be replaced by cumulative fission yields if the reactor is assumed to be at equilibirum. Fission yields from the JEFF3.3 database [6] are used in this work. Branching ratios, $\beta$ intensities, endpoint energies and spin-parity information are retrieved from the ENSDF database [7]. ENSDF data of high Q-value FP are subject to the Pandemonium effect, which is due to the partial blindness of HPGe detectors to high-energy or weak emission $\gamma$-rays, resulting in an overestimation of the $\beta$-feedings of low energy levels. Identified Pandemonium-impacted FP contribute at least to $\sim 45 \%$ of a reactor IBD yield. Their data are corrected based on data from total absorption $\gamma$-spectroscopy and from direct $\beta$ spectrum measurements. Finally, contributions of FP with no available data are estimated using an effective modeling assuming three allowed transitions with evenly distributed endpoint energies up to the maximum decay energy of the FP.

\section{Uncertainty treatment}

Fission yield uncertainties are analytically propagated and induce a $\sim 1.1 \%$ uncertainty on a reactor IBD yield. Uncertainties of branching ratios, $\beta$ intensities, endpoint energies and spin-parity information are propagated using a Monte-Carlo simulation. The former two sources induce a $\sim 0.6 \%$ IBD yield uncertainty, while the other two induce a $\sim 0.2 \%$ IBD yield uncertainty. Beside nuclear data uncertainties, spectrum mismodelings are also treated as uncertainty sources. Non-unique forbidden transitions are especially very complicated to compute, and contribute to $\sim 35 \%$ of a reactor IBD yield. Nonunique transitions of degree $N$ are approximated as unique transitions of degree $N-1$, i.e. transitions of same spin change but different parity change, which introduces modeling errors. This is called the $\xi$ -

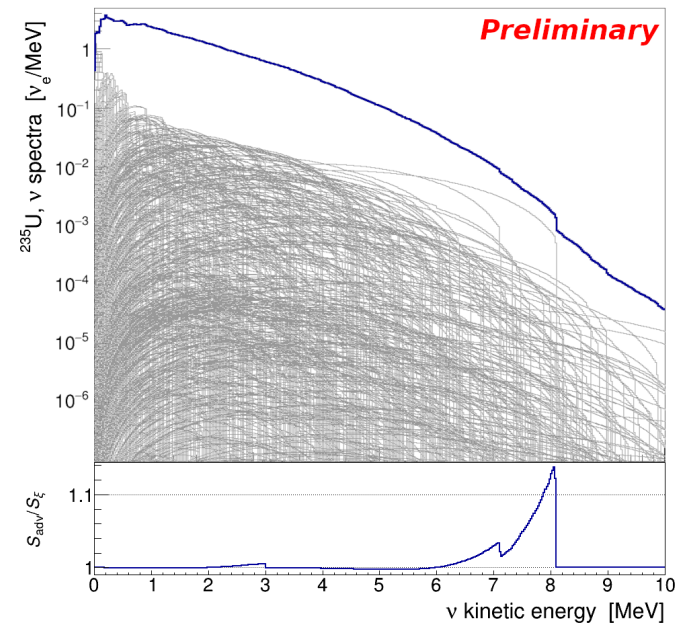

Figure 1: Top: ${ }^{235} \mathrm{U} \bar{v}_{e}$ fission spectrum (in blue) and FP spectra (in grey) composing it, obtained with the revised SM. Bottom: ratio of ${ }^{235} \mathrm{U}$ fission spectra using nuclear structure calculations $\left(S_{\text {adv }}\right)$ or the $\xi$-approximation $\left(S_{\xi}\right)$ for the three nonunique transitions of ${ }^{92} \mathrm{Rb},{ }^{96} \mathrm{Y}$ and ${ }^{144} \mathrm{Pr}$. 
approximation, and it has been shown to be abusively applied to all non-unique transitions wihtout any theoretical argument [8]. In this work, three of the most important non-unique transitions from ${ }^{92} \mathrm{Rb},{ }^{96} \mathrm{Y}$ and ${ }^{144} \mathrm{Pr}$, contributing to $\sim 10 \%$ of a reactor IBD yield, are estimated with nuclear structure calculations using the NuShellX program. The uncertainty associated to the modeling of these three branches is based on a comparison between the nuclear structure calculations and their $\xi$-approximated versions. Estimating the uncertainty associated to all other $\xi$-approximated transitions is on-going. Modeling uncertainties are derived for the radiative correction and the weak magnetism for each transition by comparing their spectra with and without the corrections. Some FP may still be uncorrected from the Pandemonium effect. An uncertainty must be derived for those FP, which is an on-going work. Lastly, an uncertainty must be associated to the contribution of FP with no decay data. This work is also in progress. The preliminary ${ }^{235} \mathrm{U}$ spectrum obtained with this new SM is shown in Fig. 1. The impact of modeling three of the main non-unique transitions with nuclear structure calculations is shown in the bottom plot, with an important effect above $6 \mathrm{MeV}$.

\section{Conclusion}

A new reactor $\bar{v}_{e}$ spectrum has been modeled with a revised SM using up-to-date nuclear data. Several corrections have been refined and an advanced calculation has been employed for three important non-unique transitions. This is a first step to bypass the $\xi$-approximation commonly used in other summation calculations. More non-unique transitions will be computed in the near future. The derivation of an uncertainty budget is also on-going. Uncertainties associated to possible Pandemonium-impacted FP, to non-unique transitions and to FP with no data treated with the effective modeling are still under investigation. Once done, a comprehensive uncertainty budget will be established, which to our knowledge has never been done in any summation calculations.

\section{References}

[1] T.A. Mueller et al., Improved predictions of reactor antineutrino spectra, Phys. Rev. C 83 (2011) 054615 .

[2] P. Huber, Determination of antineutrino spectra from nuclear reactors, Phys. Rev. C 84 (2011) 024617.

[3] G. Mention et al., Reactor antineutrino anomaly, Phys. Rev. D 83 (2011) 073006.

[4] A. Sirlin, Radiative correction to the $\bar{v}_{e}\left(v_{e}\right)$ spectrum in $\beta$ decay, Phys. Rev. D 84 (2011) 014021.

[5] A.C. Hayes et al., Systematic uncertainties in the analysis of the reactor neutrino anomaly, Phys. Rev. Lett. 112 (2014) 202501.

[6] A. Plompen et al., The joint evaluated fission and fusion nuclear data library, jeff-3.3, 07, 2020. 10.1140/epja/s10050-020-00141-9.

[7] From ENSDF database as of June 29th 2020. Version available at http://www.nndc.bnl.gov/ ensarchivals/.

[8] X. Mougeot, Reliability of usual assumptions in the calculation of beta and nu spectra, Phys. Rev. C 91 (2015) 055504. 